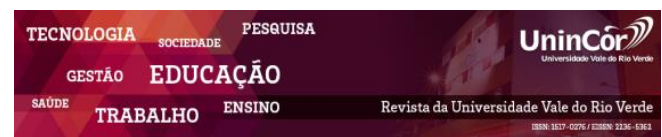

Revista da Universidade Vale do Rio Verde ISSN: 1517-0276 / EISSN: 2236-5362 Vol. $16 \mid$ n. 2 | Ano 2018

Lorena Ligia de Lima Monteiro Instituto Federal de Educação, Ciência e Tecnologia lorenaligialima@gmail.com.

Celso Lourenço de Arruda Neto Instituto Federal de Educação, Ciência e Tecnologia : celso_neto91@ @otmail.com.

\section{ESTADONUTRICIONAL E CONSUMO ALIMENTAR DE PESSOAS COM DEFICIÊNCIA INTELECTUAL}

\section{RESUMO}

Segundo a Associação Americana de Deficiência Intelectual e de Desenvolvimento - AAIDD, a deficiência intelectual é caracterizada por limitações significativas tanto em funcionamento intelectual como no comportamento adaptativo. Nos indivíduos com deficiência intelectual, observa-se uma maior prevalência de sobrepeso e obesidade. Portanto o objetivo desdetrabalho é caracterizar o estado nutricional e avaliar o consumo alimentar de deficientes intelectuais, atentando-se para a prevalência de sobrepeso e obesidade, os hábitos alimentares e de que forma as condições socioeconômicas bem como a deficiência podem estar relacionadas a tal condição. Trata-se de um estudo transversal, descritivo e de campo, realizado com portadores de deficiência intelectual do Centro Integrado de Educação Especial e Inclusão - CIEEI, no município de Morada Nova - CE. Os avaliados eram de ambos os sexos e possuíam idade entre 13 e 54 anos. Observou-se uma alta prevalência de sobrepeso e obesidade entre os participantes do estudo, sobretudo nos adultos. Este fato pode estar associado aos maus hábitos alimentares, bem como a dificuldade na aquisição de determinados alimentos devido as condições socioeconômicas. Desta forma, o estudo comprovou e consequentemente concorda com os demais existentes na literatura em que portadores de deficiência estão mais propensos a desenvolverem obesidade.

Palavras-chave:Estado Nutricional. Consumo Alimentar. DeficiênciaIntelectual. Sobrepeso. Obesidade.

\section{NUTRITIONAL STATUS AND FOOD CONSUMPTION OF PEOPLE WITH INTELLECTUAL DISABILITIES}


with poor eating habits, as well as the difficulty in acquiring certain foods due to socioeconomic conditions. Thus, the study found, and therefore agrees with others in literature in which people with disabilities are more likely to develop obesity.

Times New Roman, tamanho 10, justificado, com espaçamento simples. Parágrafo único em inglês, com cerca de 150 a 250 palavras; cinco keywords separadas por ponto e finalizadas por ponto.

Keywords:Nutritional status. Food consumption. Intellectual Disabilities. Overweight. Obesity.

Recebido em: 24/01/2018 - Aprovado em: 26/07/2018 - Disponibilizado em: 15/12/2018

\section{INTRODUÇÂO}

Segundo a Associação Americana de Deficiência Intelectual e de Desenvolvimento AAIDD, a deficiência intelectual é caracterizada por limitações significativas tanto em funcionamento intelectual como no comportamento adaptativo. Diante disso, pode-se dizer que a pessoa diagnosticadaapresenta dificuldade para aprender, entender e realizar atividades comuns para as outras pessoas (AAIDD, 2010; APAE, 2015).

O Instituto Brasileiro de Geografia e Estatística - IBGE, no censo demográfico de 2010, descreveu que $1,4 \%$ da população residente $^{1}$ no país apresenta deficiência mental ou intelectual. Identificar as causas é um grande desafio a ser enfrentado, visto que, há uma diversidade de fatores que podem levar a essa condição. Na maioria das vezes, a deficiência é resultado de uma alteração provocada por fatores genéticos, distúrbios na gestação, problemas no parto ou após o nascimento(APAE, 2015; MOURA, et al., 2012; OLIVEIRA, 2012; SCHUCK, 2012).
Problemas relacionados com a alimentação são comuns em portadores de deficiência intelectual - DI, implicando, desta forma, nos aspectos nutricionais. Dentre as dificuldades comumente encontradas estão a alteração de postura, a dessensibilização oral, os problemas gastrointestinais, a dificuldade de mastigação e deglutição, a presença de refluxos e a utilização de medicamentos (CAMPOS; GIRO; ORRICO; 2005; MOURA, et al., 2012).

Nos indivíduos com deficiência intelectual, observa-se uma maior prevalência de sobrepeso e obesidade. Estudos indicam que essa população está mais propensa a desenvolver obesidade. Dentre os fatores que levam a tal fato encontram-se: as dificuldades motoras, que comprometem o desenvolvimento de atividades físicas; assim como os maus hábitos alimentares, fruto diversas vezes da falta de informação nutricional e que podem levar a uma alta ingestão calórica (DA SILVA, SILVA, 2009; MACEDO; ESCOLBAL; GOYOS, 2013; MAUERBERGDE CASTRO, et al., 2013; QUEIROZ, 2009; RAULINO; BARROS, 2002;).

A obesidade é caracterizada pelo acúmulo excessivo de tecido adiposo, e representa um fator de risco para o surgimento de

1 População residente no país, segundo o censo de 2010 é de 45.606.048 habitantes. 
uma série de complicações, que podem afetar a qualidade de vida do indivíduo, dentre as quais estão: alteração no perfil lipídico, dificuldade respiratória, problemas cardíacos, diabetes etc. (DA SILVA; SILVA 2009; MACEDO; ESCOLBAL; GOYOS, 2013; QUEIROZ, 2009).

Diante da realidade observada, este trabalho objetiva caracterizar o estado nutricional e avaliar o consumo alimentar de deficientes intelectuais, atentando-se para a prevalência de sobrepeso e obesidade, para os hábitos alimentares, assim como de que forma as condições socioeconômicas e o quadro de deficiência podem estar relacionadas ao excesso de peso.

\section{METODOLOGIA}

Trata-se de um estudo transversal, descritivo e de campo, realizado com portadores de deficiência intelectual do Centro Integrado de Educação Especial e Inclusão - CIEEI, no município de Morada Nova - CE.

A pesquisa foi realizada após a assinatura do termo de consentimento livre e esclarecido pelos pais/responsáveis e termo de assentimento pelos alunos do referido centro, vale ressaltar que o critério de inclusão/exclusão era estar devidamente matriculado e frequentando o centro.

A coleta dos dados aconteceu na própria instituição em três etapas. Iniciou-se com uma entrevista aos pais e/ou responsáveis para coletar os dados do participante, bem como os dados socioeconômicos.

$\mathrm{Na}$ segunda etapa foi realizada uma avaliação do consumo alimentar a partir da aplicação de um questionário de frequência alimentar - QFA ao pai/mãe ou responsável, tendo em vista a impossibilidade de muitos dos participantes em responderem o questionário de maneira adequada, devido a deficiência e/ou o desconhecimento dos alimentos que compõem sua dieta. O QFA escolhido apresentava: o grupo alimentar, a frequência e o tipo de alimento. Este ainda contava com a possibilidade de inclusão de outro tipo de alimento a escolha do entrevistado.

Por último, realizou-se uma avaliação antropométrica para o diagnóstico nutricional. Dessa forma, foram aferidas as medidas de peso e estatura dos alunos. Para aferição de peso foi utilizada uma balança digital (Wiso) e para a estatura um estadiômetro portátil (Sanny).O protocolo para pesagem e aferição da estatura foi conforme descrito no manual de orientações do Sistema de Vigilância Alimentar e Nutricional SISVAN (BRASIL, 2011).

Para a realização do diagnostico nutricional, os participantes foram divididos conforme a fase do curso da vida em adolescentes e adultos. Para cada fase foram utilizados índices e parâmetros para o diagnostico nutricional, divididos em: magreza acentuada, magreza, eutrofia, sobrepeso, obesidade e obesidade grave para adolescentes (Quadro 1) e baixo peso, eutrofia, sobrepeso e obesidade para adultos (Quadro 2), conforme descrito pela organização mundial da saúde OMS.

Para os adolescentes foram utilizados os parâmetros Índice de Massa Corporal IMC/idade, cujo os pontos de corte foram baseados em escores. Enquadraram-se neste grupo participantes maiores de 10 anos e menores de 20 anos. 
Quadro 1 - Pontos de Corte IMC/Idade estabelecidos para Adolescentes.

\begin{tabular}{|c|c|}
\hline VALORES CRÍTICOS & $\begin{array}{l}\text { DIAGNÓSTICO } \\
\text { NUTRICIONAL }\end{array}$ \\
\hline$<$ Escore-z -3 & Magreza Acentuada \\
\hline 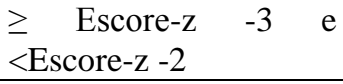 & Magreza \\
\hline $\begin{array}{l}\geq \quad \text { Escore- } \mathrm{z}-2 \quad \mathrm{e} \leq \\
\text { Escore- } \mathrm{z}+1\end{array}$ & Eutrófia \\
\hline $\begin{array}{l}\geq \quad \text { Escore }-\mathrm{Z} \quad+1 \quad \mathrm{e} \\
<\text { Escore }-\mathrm{Z}+2\end{array}$ & Sobrepeso \\
\hline $\begin{array}{l}\geq \quad \text { Escore- } \mathrm{z}+2 \text { e } \leq \\
\text { Escore- } \mathrm{z}+3\end{array}$ & Obesidade \\
\hline$>$ Escore-z +3 & Obesidade Grave \\
\hline
\end{tabular}

Fonte: (WHO, 2007)

Nota: A referência de IMC para idade da Organização Mundial da Saúde de 2007 apresenta valores até 19 anos completos, a partir desta idade a instituição considera os indivíduos como adultos. Como o Ministério da Saúde considera que a fase adulta se inicia apenas com 20 anos completos, sugere-se a adoção dos mesmos valores de 19 anos completos para a avaliação de indivíduos com 19 anos e 1 mês até 19 anos e 11 meses (BRASIL, 2011).

Para os adultos foi adotado apenas o IMC como parâmetro. Os pontos de corte estabelecidos para esta faixa etária são conforme descrito no quadro abaixo:

Quadro 2 - Pontos de Corte para Adultos.

\begin{tabular}{|l|l|}
\hline IMC $\left(\mathrm{Kg} / \mathrm{m}^{2}\right)$ & $\begin{array}{l}\text { DIAGNÓSTICO } \\
\text { NUTRICIONAL }\end{array}$ \\
\hline$<18,5$ & Baixo Peso \\
\hline$\geq 18,5<25$ & Eutrófico \\
\hline$\geq 25<30$ & Sobrepeso \\
\hline$\geq 30$ & Obesidade \\
\hline
\end{tabular}

Fonte: (WHO, 1995)

$\mathrm{O}$ projeto de pesquisa passou por apreciação e foi aprovado pelo comitê de ética do Instituto Federal de Educação, Ciência e Tecnologia, sob o parecer de $\mathrm{n}^{\circ} 2$ 2. 156.412. Os resultados obtidos da análise de todos os dados foram expressos em frequências absoluta e relativa.

\section{RESULTADOS}

\subsection{Caracterização}

Dos quarenta e um estudantes que estavam regularmente matriculados $\mathrm{e}$ frequentando o centro, trinta e um aceitaram participar da pesquisa, já os demais, os pais/responsáveis não aceitaram ou não compareceram a entrevista. Destes, $11(35,48 \%)$ eram adolescentes de ambos os sexos com idade de 13-19 anos, e 20 (64,52\%) adultos de ambos os sexos com idade de 20-54 anos.

A renda familiar dos entrevistados variou de um a três salários mínimos, sendo que alguns recebiam benefício devido a deficiência e outros não. Em relação aos entrevistados, 45,16\% $(\mathrm{n}=14)$ recebiam benefício de um salário mínimo, sendo esta a única fonte de renda familiar; 29,03\% (n=9) tinham renda de dois salários mínimos e um destes era decorrente do benefício; $16,13 \% \quad(\mathrm{n}=5)$ apresentaram renda de dois salários mínimos sem benefício e 9,68\% (n=3) possuíam três salários mínimos como renda familiar sem receberem nenhum benefício.

Quanto ao nível de instrução dos pais, constatou-se que $12,90 \%(n=4)$ eram analfabetos; $58,06 \%(\mathrm{n}=18)$ responderam ter o ensino fundamental incompleto; $16,13 \%(\mathrm{n}=5)$ ensino fundamental completo e $12,90 \%(n=4)$ ensino médio completo.

\subsection{Estado Nutricional}

Ao investigar o estado nutricional dos adolescentes baseado no IMC/Idade, observou-se que a maioria deles se encontrava na faixa de eutrofia, seguido de sobrepeso e por último obesidade grave (Gráfico 01). Nenhum dos 
participantes se enquadrou nos diagnósticos de magreza acentuada, magreza e obesidade. Os valores de sobrepeso e obesidade grave somados mostram que $45,45 \%(n=5)$ destes adolescentes estavam acima do peso.

Gráfico 1 - Estado nutricional de adolescentes com DI.

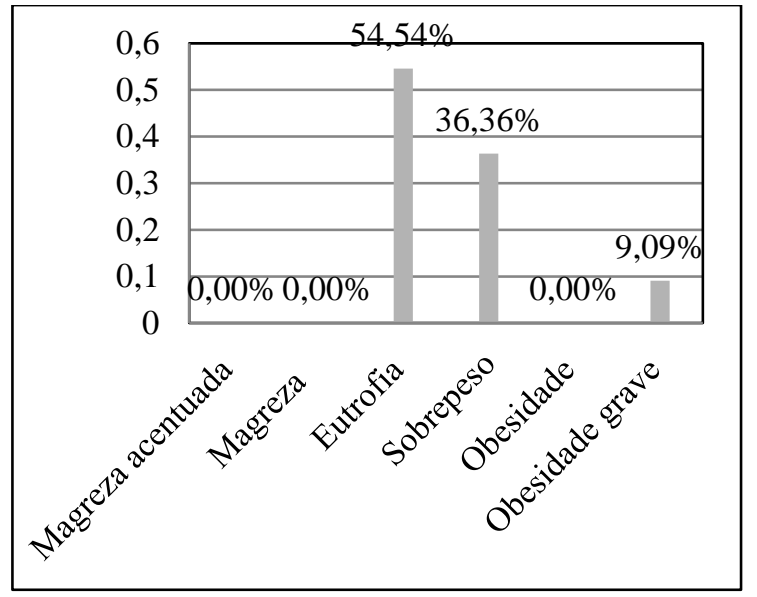

Fonte: Autores

Nos adultos, os resultados se apresentaram de forma diferenciada, baseado no parâmetro IMC, como observado no Gráfico 02, a maioria dos avaliados encontravam-se com o diagnóstico de obesidade, seguido de sobrepeso, eutrofia e baixo peso. Os resultados tornam-se ainda mais significativos quando somados os valores de sobrepeso e obesidade, totalizando $70 \%(n=14)$.

Gráfico 2 - Estado nutricional de adultos com DI.

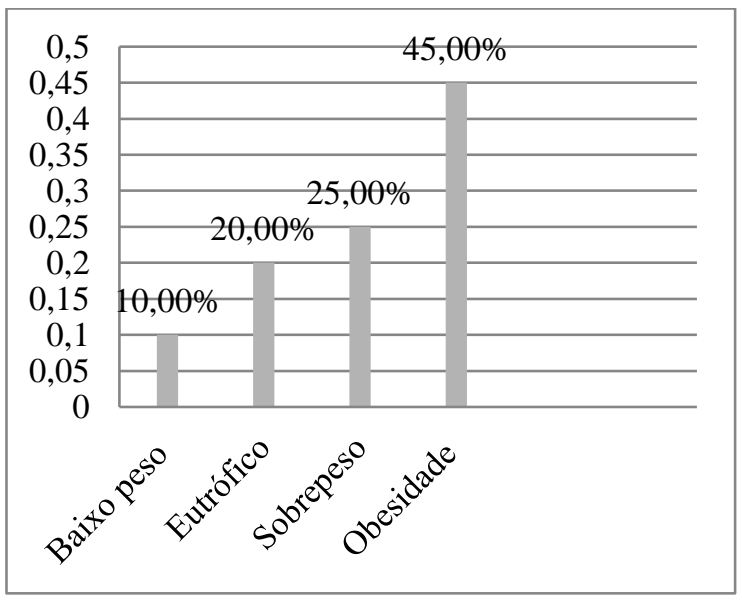

Fonte: Autores

Diante dos resultados expostos, pode-se perceber que, de acordo com a população total, a maior parte dos avaliados, 61,29\% ( $\mathrm{n}=19)$ encontravam-se entre a faixa de sobrepeso e obesidade/obesidade grave.

\subsection{Consumo Alimentar}

No que diz respeito ao consumo alimentar, a dieta dos participantes se dava basicamente pelos alimentos que estavam inseridos no questionário. Para cada grupo alimentar, obtiveram-se aqueles alimentos que eram consumidos com mais frequência (uma vez, duas a três vezes por semana ou todos os dias), os que eram menos frequentes, consumidos raramente e ainda aqueles que nunca eram consumidos pelos participantes. Logo, os dados percentuais equivalem a somatória do consumo dos alimentos semanalmente.

No grupo dos leites e derivados, destacaram-se o leite $38,71 \% \quad(n=12)$, iogurte $35,48 \%(n=11)$ e queijo $38,71 \%(n=12)$ como mais consumidos, mas não superam o elevado percentual de participantes que consomem esses alimentos raramente ou nunca, a coalhada apresentou baixa frequência de consumo. Neste grupo foi citado ainda o achocolatado como outro alimento que fazia parte da dieta dos participantes em análise.

Para o grupo das carnes e ovos, apareceram em destaque o ovo, onde 93,54\% $(n=29)$ dos avaliados consumiam este alimento pelo menos uma vez por semana. Em seguida apareceu frango $74,19 \%(\mathrm{n}=23)$, carne bovina $61,29 \% \quad(n=19)$ e peixe $35,48 \% \quad(n=11)$ respectivamente. $\mathrm{O}$ peixe, mesmo que levando em consideração o consumo do alimento uma 
vez por semana, não supera o percentual de participantes que consomem raramente ou nunca. Neste grupo foram ainda inseridos carne suína, carne ovina e embutidos.

Para as leguminosas, o feijão destacou-se dos demais e apresentou ótima frequência de consumo, 93,54\% $(n=29)$ dos avaliados. A soja, ervilha e lentilha também eram consumidas por alguns participantes, mas com frequência não relevante. Em se tratando de massas e cereais, o arroz, macarrão e pão foram os líderes deste grupo, ambos obtiveram melhor percentual entre os alimentos que eram consumidos todos os dias. Neste grupo foram inseridos pelos participantes alimentos como, farinha de mandioca, farinha de milho, biscoito e farinha de trigo.

As hortaliças e frutas estavam inseridas no mesmo grupo, as frutas apresentaram frequência relativamente boa de consumo $64,51 \%(n=20)$, todavia foi relatado por muitos pais o consumo de uma fruta específica, a banana, possivelmente por se tratar de uma fruta de fácil acesso. Os folhosos ganharam destaque nos alimentos que nunca eram consumidos pelos participantes $80,64 \%(n=25)$, quanto as verduras e legumes, um elevado percentual de participantes nunca utilizavam esses alimentos na sua dieta.

No grupo destinado às gorduras, o óleo de soja foi o líder de consumo 80,64\% $(n=25)$, consumido na maioria das vezes todos os dias, seguido da margarina cremosa que também destacou-se pelo seu elevado consumo. Os demais não apresentaram frequência relevante.

Por último, no grupo das bebidas e açúcar apresentaram destaque como mais consumidos o açúcar $80,64 \%(n=25)$ e os doces $58,07 \% \quad(n=18) . \quad O \quad$ refrigerante apresentou percentual mais elevado nos alimentos que eram consumidos de 2-3 vezes por semana, foram acrescentados a esse grupo como outros alimentos consumidos o suco natural e o refresco em pó.

\section{DISCUSSÃO}

Percebe-se uma grande carência de trabalhos desenvolvidos com portadores de deficiência intelectual no Brasil e no mundo, em especial os que abordam questões de estado nutricional e de consumo alimentar, visto que essa é uma população mais vulnerável, em que há uma maior exigência no que diz respeito aos cuidados éticos e metodológicos.

Os resultados concordam com outros estudos que revelam que pessoas com deficiência intelectual apresentam elevadas taxas de sobrepeso e obesidade e que estão mais propensas a esta condição. Os dados corroboram ainda com o estudo de Raulino e Barros, (2002), realizado com homens adultos deficientes intelectuais no Distrito Federal, em que a média do peso corporal foi maior na faixa de etária de 26-44 anos do que na faixa etária de 14-25 anos, ou seja, os jovens participantes do estudo que estão na faixa de eutrofia tem grande probabilidade de desenvolver sobrepeso e obesidade quando adultos (DA SILVA; SILVA, 2009; MARTÍN, et al., 2016; RAULINO; BARROS, 2002; ROBERTSON, et al., 2014; SOUSA, et al., 2015)

A obesidade é uma enfermidade crônica caracterizada pelo acúmulo excessivo de gordura, que além de prejudicar a locomoção, o que levaria a uma menor aptidão física, está associada ao surgimento de uma série de 
morbidades. Tendo em vista que estes pacientes já possuem suas limitações devido a deficiência, o desenvolvimento da obesidade acaba agravando ainda mais o quadro. (DA SILVA; SILVA, 2009; FISBERG, et. al., 2005; SOUSA, et al., 2015).

Os maus hábitos alimentares associados ao sedentarismo e as condições socioeconômicas podem contribuir para o desenvolvimento de obesidade nesse público. Pesquisas revelam que a atividade física em pessoas com deficiência intelectual é relativamente menor do que em seus pares sem deficiência. Tal condição se dá dentre vários aspectos a dificuldade que muitos encontram quanto a locomoção devido a própria deficiência (MARTÍN, et al., 2016; ROBERTSON, et al., 2014).

Segundo Da Silva e Silva (2005), ao fazerem uma associação entre o índice de massa corporal (IMC) e os hábitos alimentares em um estudo com deficientes intelectuais de uma escola especial em São José dos Campos-SC, sugeriram que indivíduos que estão fora da faixa recomendável do IMC apresentam, em sua maioria, hábitos alimentares ruins. Uma boa alimentação torna-se portanto crucial para a prevenção do quadro de obesidade e patologias associadas.

Atentando-se para os hábitos alimentares da população estudada, observa-se a partir do questionário aplicado os maus hábitos alimentares, com dificuldade de inserção de alimentos importantes como, legumes, frutas, hortaliças dentre outros, tendo em contrapartida um alto consumo de alimentos calóricos e que não trazem tantos benefícios como, os óleos, açucares e doces. Exceto as massas e cereais mais comuns e o feijão, os demais alimentos apresentaram um consumo bem diferenciado. É válido ressaltar que os alimentos de um grupo não devem ser substituídos por outro de um grupo distinto, todos são essenciais e necessários para o bom funcionamento do organismo (CAMPOS; GIRO; ORRICO, 2005; DA SILVA; SILVA, 2005; FISBERG, et. al., 2005).

$\mathrm{O}$ leite e seus derivados constituem um grupo de alimentos de grande valor nutricional, fonte de proteínas, vitaminas e minerais. O consumo desse alimento se faz necessário em especial para que se atinja a recomendação diária de cálcio, que dentre tantos benefícios, é responsável por funções estruturais e funcionais que auxiliam na formação e manutenção do esqueleto. Para a população saudável, é recomendado o consumo de três porções diárias de leite e derivados para que as recomendações de proteínas e cálcio sejam atingidas. No estudo, observou-se um baixo consumo diário desses alimentos, que pode está ligada a escolaridade e renda familiar, como sugeri a última pesquisa de orçamento familiar 2008-2009, que indica que quanto maior a renda familiar e escolaridade, maior o consumo de leite e derivados (AMANCIO, et. al., 2015).

O baixo consumo de carne bovina no grupo das carnes e ovos foi atribuído as condições econômicas relatadas pelos entrevistados. O ovo além de apresentar um custo mais baixo é uma excelente fonte de proteína, vitaminas e minerais, todavia é necessário atentar-se para a quantidade de colesterol existente no alimento que pode tornar seu consumo um pouco restritivo. A forma de preparo também deve ser levado em consideração, tendo em vista que na maioria das vezes o ovo é consumido frito, utilizando uma 
outra fonte de gordura elevando assim as calorias e consequentemente contribuindo para o quadro de sobrepeso e obesidade. Isso torna-se preocupante, tendo em vista que a maioria dos avaliados preferem este alimento. Em contrapartida, o peixe, alimento com baixo teor de gordura, alto teor proteico e fonte de ácidos graxos poli-insaturados, apresentou baixo consumo, o que é preocupante, tendo em vista que estudos revelam que o alto consumo de peixe está relacionado ao menor risco de desenvolvimento de doenças cardiovasculares (SANTOS et al, 2015; SARTORI; AMANCIO, 2012).

O desconhecimento e a indisponibilidade de outros alimentos, fizeram do feijão o alimento mais consumido no grupo das leguminosas, apresentando consumo significativamente bom entre os participantes. As leguminosas são fontes de proteína, fibras, vitaminas e minerais. O grupo das massas e cereais, principais alimentos fonte de carboidratos, apresentaram boa aceitação pelo público estudado. É importante ressaltar que não houve relato de consumo de cereais integrais, importante fonte de fibra que auxilia no bom funcionamento intestinal. A mistura arroz e feijão traduz de forma fidedigna a realidade alimentar da maioria dos brasileiros (BRASIL, 2014).

Diversos estudos e diretrizes apresentam o comportamento alimentar e o padrão de atividade física como essenciais para prevenção ou controle do excesso de peso, objetivando ainda que mudanças primordiais poderiam ser realizadas para redução ou controle do excesso de peso para essa população especifica. Zorzo, (2015), relata que a preocupação com a nutrição para o adequado desenvolvimento da saúde física e mental deve ser constante e iniciada desde antes da concepção e seguir em todas as etapas da vida devendo o indivíduo receber orientação e educação nutricional da família e também da escola (CASEY; RASMUSSEN, 2013; SPANOS; MELVILLE; HANKEY; 2013).

\section{CONCLUSÃO}

Observou-se a prevalência de sobrepeso e obesidade no grupo estudado, associados a maus hábitos alimentares e condições socioeconômicas que limitam a aquisição de alimentos importantes para a dieta dos mesmos. Não houve relatos de dificuldades relacionadas a própria deficiência, como dificuldade de mastigação e deglutição. Desta forma, o estudo concorda com os demais existentes na literatura, em que pessoas com deficiência intelectual estão mais propensos a desenvolverem obesidade. Mais estudos, principalmente no Brasil, devem ser realizados.

\section{REFERÊNCIAS}

AAIDD, Intellectual Disability: Definition, Classification, and Systems of Supports, $11^{\text {a }}$ Edição, 2010. In:AMERICAN ASSOCIATION ON INTELLECTUAL AND DEVELOPMENTAL DISABILITIES (AAIDD).Disponívelem: <http://aaidd.org/intellectual-disability/definition>. Acesso em: 07 jul. 2015, 9:30:00.

AMANCIO O. M. S. et al. Sociedade Brasileira de Alimentação e Nutrição - SBAN, A importância do consumo de leite no atual cenário nutricional brasileiro, 2015.

APAE DE SÃO PAULO. Sobre a deficiência Intelectual - o que é, 2015. Disponível em: <http://www.apaesp.org.br/SobreADeficienciaIntelect ual/Paginas/O-que-e.aspx>. Acesso em: 07 jul. 2015, 19:30:00. 
BRASIL. Ministério da Saúde. Secretaria de Atenção à Saúde. Departamento de Atenção Básica. Guia alimentar para a população brasileira / Ministério da Saúde, Secretaria de Atenção à Saúde, Departamento de Atenção Básica. - 2. ed. - Brasília: Ministério da Saúde, 2014. 156 p. : il.

Ministério da Saúde. Secretaria de Atenção à Saúde. Departamento de Atenção Básica. Orientações para a coleta e análise de dados antropométricos em serviços de saúde: Norma Técnica do Sistema de Vigilância Alimentar e Nutricional - SISVAN / Ministério da Saúde, Secretaria de Atenção à Saúde, Departamento de Atenção Básica. Brasília: Ministério da Saúde, 2011.76 p.: il. - (Série G. Estatística e Informação em Saúde).

CAMPOS, J. A. D. B.; GIRO, E. M. A.; ORRICO, S. R. P. Comparação do padrão de alimentação de portadores com necessidades especiais institucionalizados e não institucionalizados. AlimNutr, Araraquara, v. 16, n. 3, p. 273-277, jul./set. 2005.

CASEY A. E.; RASMUSSEN R. Reduction measures and percent body fat in individuals with intelectual disabilities: A scoping review. Disabilityand Health jornal, Antigonish, NS, Canada, v. 6, p. 2-7, 2013.

DA SILVA, R.; SILVA, G. P. Características antropométricas e nutricionais de pessoas com deficiência mental. Fit Perf J, Rio de Janeiro, v. 8, n. 2, p. 130-135, 2009.

DE ONIS, M. et al. Development of a WHO growth reference for school-aged children and adolescents. Bulletin of the World Health Organization, [S. 1.], v. 85 , p. 660-667, 2007.

FISBERG, R. M.; VILLAR, B. S.; COLUCCI, A. C. A.; PHILIPPI, S. T. Alimentação Equilibrada na Promoção da Saúde. In. CUPPARI L. (2. Ed. Ver. E ampl.). Nutrição clínica no adulto - Guia de medicina ambulatorial e hospitalar. Barueri, SP: Nestor Schor, p. 63-70, 2005

MACEDO, M.; ESCOBAL, G.; GOYOS C. Escolha e preferência por alimentos com ou sem valor calórico em crianças com deficiência intelectual e sobrepeso. Acta Comportamentalia, v. 21, n. 1, p. 83-98, 2013.

MARTÍN, I. S. M. et al. Análisdel estado nutricional y composición corporal de personas condiscapacidad intelectual. RevNeurol, n. 62, p. 493-501, 2016.

MAUERBERG-DE CASTRO, ET AL. Educação física adaptativa inclusiva: impacto na aptidão física de pessoas com deficiência intelectual. Revista Ciência em Extensão, v.9, n.1, p.35-61, 2013.

MOURA, A. B.; ANDREICO, A. P.; PAGANOTTO, M.; PASSONI, C. R. M. S.M. Perfil nutricional de crianças e adolescentes com deficiência intelectual.
Pediatria Moderna, Moreira Jr. Editora, v. 48, n. 8, 2012.

OLIVEIRA, L. M. B.; Secretaria de Direitos Humanos da Presidência da República - SDH/PR; Secretaria Nacional de Promoção dos Direitos da Pessoa com Deficiência - SNPD; Coordenação-geral do sistema de informações sobre a pessoa com deficiência. Cartilha do Censo 2010 - Pessoas com Deficiência. Brasília, Ed. $1^{\text {a }}$, 2012. 32p.

QUEIROZ, C. R. L. Facilidades e dificuldades à adesão de adolescentes obesos com deficiência intelectual e suas famílias, para cumprir programa de orientação e prevenção das complicações da obesidade [Dissertação de Mestrado]. São Paulo: Faculdade de saúde pública da USP, 2009.

RAULINO, A. G. D.; BARROS, J. F. Estudo do comportamento da composição corporal em homens portadores de deficiência mental no distrito federal. Rev. Bras. Ciên. e Mov., Brasília, v. 10, n. 4, p. 63 70, out. 2002.

ROBERTSON, J.; EMERSON, E.; BAINES, S.; HATTON C. Obesity and health behaviours of British adults with self-reported intellectual impairments: cross sectional survey.BMC publichealth, 14:219, 2014.

SANTOS R. D. et al. Sociedade Brasileira de Cardiologia. I Diretriz sobre o consumo de Gorduras e Saúde Cardiovascular. ArqBrasCardiol. p. 1-40, 2013.

SARTORI, A. G.; AMANCIO, R. D. Pescado: importância nutricional e consumo no Brasil. Segurança Alimentar e Nutricional, Campinas, v. 19, n. 2, p. 83-93, 2012.

SCHUCK, P. F. Fenilcetonúria como causa de Deficiência Intelectual. Revista DI, v. 2, n. 2, p. 1419, 2012.

SOUSA, G. R.; PINTO, M. G.; SEEBER J. R.; SILVA, D. A. S. Associação do estado nutricional com aptidão física relacionada a saúde em adultos com deficiência intelectual. RevBrasEducFís Esporte, São Paulo, v. 29, n. 4, p. 543-550, 2015.

SPANOS, D.; MELVILLE, C. A.; HANKEY, C. R. Weight management interventions in adults with intellectual disabilities and obesity: a systematic review of the evidence. Nutrition Journal, 12:132, 2013.

WORLD HEALTH ORGANIZATION.Physical status: the use and interpretation of anthropometry. Geneva, Switzerland: WHO, 1995. (WHO TechnicalReport Series, n. 854).

ZORZO, R. A. A importância de uma alimentação saudável para um desenvolvimento físico e mental 
adequados. Pediatria Moderna, Moreira Jr. Editora, v. 15, n. 8, p. 293-288, 2015.

\section{Lorena Ligia de Lima Monteiro}

Nutricionista - Instituto Federal de Educação, Ciência e Tecnologia, Especializanda em Gestão em Saúde Universidade da Integração Internacional da Lusofonia Afro- Brasileira, Mestranda em Tecnologia de Alimentos Instituto Federal de Educação, Ciência e Tecnologia. Email:

\section{Celso Lourenço de Arruda Neto}

Nutricionista - Instituto Federal de Educação, Ciência e Tecnologia, Especialista em Gestão em Saúde Universidade da Integração Internacional da Lusofonia Afro-Brasileira, Mestre em Saúde e Sociedade Universidade Estadual do Rio Grande do Norte. 